\title{
Nitschke, Paula: Digitalisierung auf der Mesoebene: Die Onlinekommunikation von Interessenorganisationen als Institutionalisierung
}

\author{
Oehmer, Franziska
}

\begin{abstract}
Der kommunikationswissenschaftliche Forschungskorpus zur Bedeutung von Digitalisierungsprozessen vor allem in Zeiten von Wahlen ist mittlerweile beträchtlich. Vergleichsweise wenig Beachtung fanden hingegen Digitalisierungsfolgen von kollektiven Akteuren. Paula Nitschke richtet in ihrer Dissertation den Fokus auf die Onlinekommunikation von Interessenorganisationen und verhilft damit sowohl der Mesoebene als vernachlässigter Analyseperspektive als auch einem wenig beachteten intermediären Akteur zu Aufmerksamkeit. Ihr Interesse gilt den „langfristigen Reaktionen [...], durch welche Onlinekommunikation zum dauerhaften Bestandteil organisationalen Handelns und organisationaler Strukturen wird“ (S. 3). Sie will somit Kommunikationshandeln in Routinezeiten abbilden und organisationssoziologisch erklären. Das vornehmliche Ziel der Arbeit stellt entsprechend „die Entwicklung einer theoretischen Konzeption dar, mit der der thematische Komplex aus Onlinekommunikation, Interessenorganisationen und Institutionalisierung theoretisiert werden kann“ (S. 6). Die daran anschließenden Fallstudien dienen dann der Plausibilisierung des Konzepts.
\end{abstract}

DOI: https://doi.org/10.1007/s11616-020-00583-7

Posted at the Zurich Open Repository and Archive, University of Zurich ZORA URL: https://doi.org/10.5167/uzh-198799

Journal Article

Accepted Version

Originally published at:

Oehmer, Franziska (2020). Nitschke, Paula: Digitalisierung auf der Mesoebene: Die Onlinekommunikation von Interessenorganisationen als Institutionalisierung. Publizistik, 65:485-487.

DOI: https://doi.org/10.1007/s11616-020-00583-7 


\section{Rezension}

zu Nitschke, P. (2019). Digitalisierung auf der Mesoebene. Die Onlinekommunikation von Interessenorganisationen als Institutionalisierung. Wiesbaden: Springer.

von Franziska Oehmer

Zeichen: 6405

Der kommunikationswissenschaftliche Forschungskorpus zur Bedeutung von Digitalisierungsprozessen für Individuen und Gesellschaften vor allem in Zeiten von Wahlen ist mittlerweile beträchtlich. Vergleichsweise wenig Beachtung fanden hingegen Digitalisierungsfolgen von kollektiven Akteuren. Paula Nitschke richtet in ihrer Dissertation den Fokus auf die Onlinekommunikation von Interessenorganisationen und verhilft damit sowohl der Mesoebene als vernachlässigte Analyseperspektive als auch einem wenig beachteten intermediären Akteur zu Aufmerksamkeit. Ihr Interesse gilt dabei konkret den „langfristigen Reaktionen [...], durch welche Onlinekommunikation zum dauerhaften Bestandteil organisationalen Handelns und organisationaler Strukturen wird" (S.3). Sie ist damit in der Lage, Kommunikationshandeln in Routinezeiten abzubilden und organisationssoziologisch zu erklären. Anders als ein erster Blick auf die Gliederung der Arbeit erwarten ließe, stellt das vornehmliche Ziel der Arbeit „die Entwicklung einer theoretischen Konzeption dar, mit der der thematische Komplex aus Onlinekommunikation, Interessenorganisationen und Institutionalisierung theoretisiert werden kann“ (S.6). Die daran anschließenden Fallstudien dienen dann der Plausibilisierung des Konzepts.

Die Gretchenfrage der Interessenorganisationsforschung ist die Frage nach der Definition dieses heterogenen Akteurstyps. Nitschke adressiert diesen Punkt bereits (ein wenig versteckt) in der Einleitung ihrer Arbeit: Sie versteht, zusammengefasst, Interessenorganisationen als Organisationen, die sich dauerhaft und mithilfe einer ausdifferenzierten Organisationstruktur für die Durchsetzung gemeinsamer Interessen einsetzen (siehe Definition S.5). Sie wählt damit eine sehr breite Akteursdefinition, die grundsätzlich auch einige Stiftungen, Kirchen oder auch Kammern einschließen würde. Diese können sich jedoch mit Blick auf ihre Ziele, Beziehung zur Umwelt und anderen Organisationen und damit auch auf ihre „Freiheitsgrade“ bei der Wahl und Implementation von Kommunikationsstrategien und -repertoires erheblich unterscheiden.

Zunächst referiert und diskutiert Nitschke den Forschungsstand zur Onlinekommunikation. Sie berücksichtigt dabei eine Vielzahl an Forschungszweigen: Insgesamt differenziert sie zwischen einer normativ-orientierten Debatte in der politischen Kommunikationsforschung, der instrumentellen Debatte in der PR-orientierten Forschung sowie der organisationssoziologischen Debatte. Während sie sich von den ersten beiden explizit abgrenzt (aber dennoch umfassend darstellt), bildet die dritte Debatte die Grundlage ihrer Argumentation. Forschung, die sich explizit mit ihrem Analysegegenstand - der (Online)kommunikation von Interessenorganisationen widmet, kommt dabei leider - aufgrund der sehr breit gewählten Perspektive fast zwangsläufig - zu kurz. 
Kern der Arbeit stellt die Entwicklung des theoretischen Konzeptes zur Analyse von Digitalisierungsprozessen auf der Mesoebene dar (Kap. 3 - 5). Als theoretisches Rahmengerüst stützt sich Nitschke auf den Neoinstitutionalismus. Sie reiht sich damit mit einer bedeutsamen und prägenden Arbeit ein in den Reigen der größer werdenden Forschergemeinde, die gerade in den vergangenen Jahren diese Theorieperspektive als fruchtbares Analyseinstrument für die Kommunikationswissenschaft erkannt hat. Onlinekommunkation wird demzufolge als „soziale Praktik verstanden [werden], die in Interessenorganisationen im Zuge eines Institutionalisierungsprozesses durch handelnde Akteure kommunikativ konstruiert wird (S.43-44).

Zur Operationalisierung dieses Institutionalisierungsprozesses entwickelt Nitschke eine vierdimensionale Forschungsheuristik (Kap. 4), die auf einer Kombination der Institutionenkonzeption von Scott und Giddens' Strukturationstheorie basiert. Sie unterscheidet zwischen 1) Artefakten, 2) Arbeitsroutinen und -regeln, 3) Beziehungsnetzwerken und ihrer Koordinationsweise und 4) sprachbasierte Sinnwelten der Onlinekommunikation (siehe S.53). Sie berücksichtigt und modelliert dabei auch Kontextfaktoren (Kap. 5), die als Moderatoren einen Einfluss auf den Institutionalisierungsprozess ausüben (können): a) konkurrierende Handlungslogiken in der politischen Sphäre, b) ausdifferenzierte Organisationsstruktur und interne Politisierung sowie c) Digitalisierung, Datafizierung und Quantitative Sensemaking. Sie greift hierfür auf zahlreiche, sehr heterogene und umfassend erörterte Konzepte unterschiedlicher Disziplinen zurück.

Die theoretischen Erörterungen werden im Kapitel 6 zusammengefasst und genutzt, um weitere Subfragestellungen für die folgende Fallanalyse abzuleiten. Überraschend ist dabei, dass die vier Dimensionen der Forschungsheuristik nicht explizit mit den Kontextfaktoren in Zusammenhang gebracht werden. Welche Bedeutung diese Faktoren (im Zusammenspiel) für die Ausgestaltung der Onlinekommunikation haben (können), wird nicht beispielsweise durch (Hypo)thesen, die spezifische Akteursmerkmale (Organisationsform, Finanzierungsform, ...) berücksichtigen, explizit gemacht.

Im Anschluss folgen zwei „explorativ vertiefende Fallstudie[n] mit deskriptivem Charakter" (S.93) im Mehrmethodendesign mit einem Schwerpunkt auf der im Fach selten genutzten Methode der Beobachtung. Unerwartet ist hier, dass bei der Auswahl der Interessenorganisationen und auch bei der Konzeption der Analyseinstrumente (Interviewleitfaden, Beobachtungsbogen), die sehr elaborierten theoretischen Überlegungen des ersten Teils keine Rolle zu spielen scheinen. So schafft es die Arbeit leider nicht, eine Operationalisierung der theoretischen Konzeption im engeren Sinne anzubieten. Das und die leider etwas zu kurz kommende Berücksichtigung spezifischer Literatur zur Verbands- und Interessengruppenforschung aus denen konkrete Thesen zu Wirk- und Interdependenzprozessen hätten formuliert werden können, sind die einzigen Wermutstropfen.

Insgesamt überzeugt die Arbeit durch die sehr ausführlichen und interdisziplinären theoretischen Ausführungen, die organisationale und handlungsbezogene Institutionalisierungsprozesse in der Onlinekommunikation auf der Mesoebene zu erklären vermögen. Der Fokus liegt dabei auf den langfristigen strukturellen Anpassungsleistungen, die diese erst ermöglichen oder behindern. Insofern 
leistet die Arbeit einen wichtigen Erkenntnisgewinn für die Sozial- und Kommunikationswissenschaft und die Kommunikationspraxis.

\section{biographische Kurznotiz}

Dr. phil. Franziska Oehmer, Blaw ist wissenschaftlichen Mitarbeiterin am Forschungszentrum Öffentlichkeit und Gesellschaft der Universität Zürich.

Dr. phil. Franziska Oehmer, Blaw

fög - Forschungszentrum Öffentlichkeit und Gesellschaft

E-Mail: franziska.oehmer@ foeg.uzh.ch

@ rechtgutgesagt

www.foeg.uzh.ch 